\title{
On the thermodynamics of MHD wave-heated solar wind protons
}

\author{
H. J. Fahr ${ }^{1}$ and I. V. Chashei ${ }^{2}$ \\ 1 Institut für Astrophysik und Extraterrestrische Forschung, Universität Bonn, Auf dem Hügel 71, 53121 Bonn, Germany \\ 2 Lebedev Physical Institute, Leninskii pr.53, 117924 Moscow, Russia
}

Received 17 May 2002 / Accepted 23 August 2002

\begin{abstract}
It has been clearly observed by the NASA deep-space probes that the solar wind protons do not adiabatically cool as expected towards larger solar distances, but appear to be heated by non-collisional energy sources. In some papers these heating sources were directly or indirectly ascribed to pick-up ions incorporated as suprathermal ions into the background solar wind. Neutral interstellar H-atoms penetrate into the inner heliosphere and at ionization they are converted into pick-up ions. Here we do not consider how the magnetized solar wind flow incorporates these ions into the plasma bulk when enforcing their co-motion. We simply take the first step of their incorporation for guaranteed, namely the fast redistribution of pickups from an initially unstable toroidal to a quasistable bi-spherical distribution. The free energy lost by pick-ups during this redistribution goes into the turbulent MHD waves, and as such cascades down to the proton dissipation scale and finally is absorbed by solar wind protons. Here we investigate the thermodynamics of solar wind protons being heated by absorption of this free energy of pick-ups. In addition we also consider as a relevant and competing proton heat source the heating due to absorption of wave energy of convected MHD turbulences, showing that the latter source always dominates inside some critical solar distance, whereas the first one dominates in the outer heliospheric regions. We then solve the resulting differential equation for the solar wind proton temperature and show in the solutions obtained that a quasipolytropic behaviour of the solar wind protons with a distance-dependent polytropic index is found. The expression for the pressure clearly shows the change from an adiabatic to a quasipolytropic behaviour with a decreasing polytropic index at increasing distances as observed by the VOYAGERs. The quantitative run of the temperature and the polytropic index with solar distance thereby is strongly influenced by the interstellar H-atom density. The (pick-up ion)-induced heating also evidently leads to a wind-asymmetric solar wind temperature distribution with higher temperatures occuring in upwind direction compared to downwind direction.
\end{abstract}

Key words. Sun: solar wind - Sun: particle emission - plasmas

\section{Introduction to the dynamics of the distant solar wind}

It has for a long time been clearly recognized that the solar wind dynamics at larger distances is influenced by the creation and incorporation of pick-up ions. First we briefly consider this pick-up ion-induced modulation effect. It has been predicted already quite early that the solar wind becomes decelerated due to both the pick-up ion loading (see e.g. Holzer \& Leer 1973; Fahr 1973; Ripken \& Fahr 1983; Fahr \& Ripken 1984) and, more recently, also due to the action of the pick-up ion pressure (Fahr \& Fichtner 1995; Lee 1997; Whang 1998; Whang et al. 1999; Fahr \& Rucinski 1999). While being decelerated the solar wind plasma is also heated, partly because of adiabatic compression with respect to a non-decelerated flow, partly because of being loaded with suprathermal pick-up ions. As an overall effect the effective solar wind sound velocity is increased (see

Send offprint requests to: H. J. Fahr,

e-mail: hfahr@astro.uni-bonn.de
Fahr \& Rucinski 1999, 2001). Connected to both phenomena, i.e. deceleration and heating, the solar wind Mach numbers also decrease with increasing solar distances. We shall only briefly review these phenomena here.

The effective Mach number and the wind deceleration is calculated with the help of an expression for the pick-up pressure, $P_{i}$. The dynamical effect of this pressure has, however, not yet been taken into account in a fully consistent form since it would need the simultaneous and consistent treatment of the solar wind plasma, the pick-up ions and the involved MHD waves. Semi-consistent solutions of a kinetic pick-up ion transport equation prescribing the solar wind velocity profile have, however, meanwhile been presented (see e.g. Chalov et al. 1995, 1997; Fichtner et al. 1996) which also permit the calculation of the pick-up ion pressure as a velocity moment of the pick-up ion distribution function. Representing this pickup pressure in the form $P_{i}=\alpha \rho_{i} V_{w}^{2}$ where $\alpha$ represents a pure function of the solar distance $r$, and where $\rho_{i}$ and $V_{w}$ are the pick-up ion mass density and the solar wind bulk velocity, 
respectively, one then can derive the following differential equation for the decelerated solar wind (see Fahr \& Rucinski 2001):

$\frac{\mathrm{d}}{\mathrm{d} r} V_{w}=\frac{-m_{\mathrm{p}} \beta_{\mathrm{ex}} \frac{1+\alpha}{\rho_{w}+\rho_{i}}+\frac{2 \alpha}{r} \xi V_{w}}{1+\alpha \xi}$.

Here $\beta_{\text {ex }}$ denotes the local injection rate of pick-up ions which is assumed to be exclusively due to charge exchange processes with protons (photoionization is neglected) and thus is given by $\beta_{\mathrm{ex}}=\sigma_{\mathrm{ex}} n_{\mathrm{H}} n_{w} V_{w}$. The function $\xi=\rho_{i} /\left(\rho_{w}+\rho_{i}\right)$ denotes the relative abundance of pick-ups with respect to all protons and is adopted here as was calculated by Fahr \& Rucinski (1999) using the "hot" kinetic H-atom model developed by Wu \& Judge (1979). The integration of the differential Eq. (1) then yields:

$V_{w}=V_{w 0} \exp \left[\int_{r_{0}}^{r} \frac{\frac{2 \alpha}{r} \xi-n_{\mathrm{H}} \sigma_{\mathrm{ex}}(1-\xi)(1+\alpha)}{1+\alpha \xi} \mathrm{d} r\right]$.

A fairly realistic expression for $P_{i}$ was derived from results for $f_{i}$ obtained by Chalov et al. $(1995,1997)$ as solutions of the complete pick-up ion transport equation. As Fahr \& Lay (2000) showed these numerical results can be represented by the following analytical formula:

$f_{\text {pui }}=\digamma\left(x^{-0.33}\right) w^{\beta} \exp \left[-C(x)\left(w-w_{0}\right)^{\kappa}\right]$,

where $\digamma$ is a constant, $x=r / r_{0}$ is the radial solar distance in units of $r_{0}=1 \mathrm{AU}, w=\left(v / V_{w}\right)^{2}$ is the squared pick-up ion velocity normalized with $V_{w}, w_{0}$ being a typical injection value. Furthermore it was found: $\beta=-\frac{1}{6} ; \kappa=\frac{2}{3}$; and: $C(x)=$ $0.442 x^{0.2}$. As shown in Fahr \& Rucinski (2001) with the above representation of $f_{i}$ one obtains the following representation of $P_{i}$ :

$P_{i}=\frac{5}{16} \sqrt[2]{\pi} C(x)^{-\frac{3}{2}} \rho_{i} V_{w}^{2}=\alpha(x) \rho_{i} V_{w}^{2}$.

In this expression for $P_{i}(x)$ the function $\alpha$ is found as given by: $\alpha=\alpha(x)=1.83 x^{-0.3}$. This means that $\alpha$ decreases slightly with increasing solar distances, obviously reflecting the fact that at larger distances the adiabatic deceleration starts to slowly overcompensate the effect of wave-driven Fermi-2 accelerations. It is, however, interesting to see that even without Fermi-2 accelerations (i.e. resulting for very low turbulence levels) the ratio of $P_{i} / \rho_{i}$ also turns out to be nearly constant (see Vasyliunas \& Siscoe 1976), its magnitude only being lower.

The above formula is valid at distances of $x \geq x_{\mathrm{c}}=15$ where $\alpha=\alpha_{\mathrm{c}}=\alpha\left(x_{\mathrm{c}}\right)$ equal to $\alpha_{\mathrm{c}}=0.44$. In the following calculations we assume that $P_{i}$ can be represented with sufficient accuracy by Eq. (4) setting $\alpha(x)=\alpha_{\mathrm{c}}$.

If we then calculate the solar wind deceleration with Eq. (2), after expansion of the exponential for small arguments, the relative deceleration of the solar wind $\Delta X=$ $\left(V_{w 0}-V_{w}\right) / V_{w 0}$ given by:

$\Delta X(r)=\int_{r_{0}}^{r} \frac{\frac{2 \alpha_{\mathrm{c}}}{r} \xi-n_{\mathrm{H}} \sigma_{\mathrm{ex}}(1-\xi)\left(1+\alpha_{\mathrm{c}}\right)}{1+\alpha_{\mathrm{c}} \xi} \mathrm{d} r$

depending on the interstellar $\mathrm{H}$-atom density $n_{\mathrm{H} \infty}$ and the solar distance $r$ is always in the range of $\Delta X \leq 0.1$. For instance approximating $\xi$ by (see Fahr \& Rucinski 1999): $\xi(r) \simeq \sigma_{\mathrm{ex}} \int_{r_{0}}^{r} n_{\mathrm{H}}\left(r^{\prime}\right) \mathrm{d} r^{\prime}$ using formula (5) and adopting the LISM H-atom density with $n_{\mathrm{H} \infty}=0.1 \mathrm{~cm}^{-3}$, then leads one to a value of $\Delta X(40 \mathrm{AU})=0.05$ or $\Delta X(60 \mathrm{AU})=0.07$. On the basis of these derivations we may conclude that the solar wind velocity $V_{w}$ within $r \leq 80 \mathrm{AU}$ can be taken as constant and given by $V_{w} \simeq V_{w 0}$.

\section{Heating sources for the solar wind protons}

Here we want to consider heat sources acting upon solar wind protons at their propagation to large solar distances. A direct heating of solar wind protons by the keV-energetic pick-up ions embedded in the solar wind via Coulomb collisions can safely be excluded at distances beyond $0.3 \mathrm{AU}$. The only immaginable and available mechanism to heat solar wind protons is by dissipation of MHD turbulent wave energy. This was already suggested by Parker (1964) and Coleman (1968) who had predicted that some extended heating due to dissipation of waves might cause a non-adiabatic expansion of the solar wind to regions beyond its critical point. Further detailed studies of the coronal proton temperature and temperature anisotropy variation with solar distance due to ion heating by resonant waveparticle interaction with coronal ion-cyclotron and fast magnetosonic waves were carried out by Marsch et al. (1982). These studies were later extended to more general conditions through quasilinear plasma-wave simulation calculations by Liewer et al. (2001) and Cranmer (2001) and they all could clearly show that non-adiabatic expansion of the solar wind protons has to be expected.

This non-adiabatic solar wind temperature behaviour meanwhile is manifest in data taken by the VOYAGER-1/2 spacecraft up to large solar distances (i.e. $r \geq 20 \mathrm{AU}$ ) (see Richardson et al. 1995; Whang 1998; Whang et al. 1999). A more quantitative study of the dissipation of non-Alfvénic turbulence energy to solar wind protons even at larger solar distances was carried out by Matthaeus et al. (1994) with the result that wave energy is absorbed with a rate $q_{\mathrm{turb}} \simeq \rho_{\mathrm{s}} u^{3} / l$ where $\rho_{\mathrm{s}}, u, l$ are the solar wind mass density, the rms turbulent fluctuation speed, and the turbulent correlation scale. The dependence of these quantities $u$ and $l$ on distance $r$ was investigated by Zank et al. (1996) who described the evolution of low-frequency turbulence power in the solar wind on the basis of a scale-separated equation developed by Zhou \& Matthaeus (1990) and describing the evolution of amplitude fluctuations $u$ and $b$ about the mean velocity $V_{w}$ and the mean magnetic field $B$. In this equation for the frequency-averaged fluctuation power these authors took into account nonlinear dissipation terms and power sources. Amongst the latter they discussed terms due to wave-driving by velocity shears and compressional effects associated with solar wind interaction regions and due to pick-up ions which after their injection populate a distribution function which is unstable with respect to turbulence excitation. In the solutions obtained for $u^{2}(r)$ and $l(r)$ they could demonstrate that the usual WKB approximations are far from what realistically can be expected in the solar wind at large distances. Far away from solar wind interaction regions and at higher heliographic latitudes one should anyway not expect to 
find shear-induced turbulent energy, but outside of the so-called ionization cavity one should find pick-up ion induced turbulent energy and correlation lengths $l(r)$ which from 5 AU outwards systematically decrease with distance.

On the basis of these results Smith et al. (2001) also analysed the heating of the distant solar wind due to absorption of wave turbulent energy by protons, solving a system of coupled differential equations which describe the evolution with distance of the mean turbulent energy $u^{2}$, the correlation length $l$, and the proton temperature. The nonlinear dissipative loss term in the equation for $u^{2}$ was at the same time taken with the opposite sign, as an energy gain term for the protons. Comparison of results with VOYAGER data seem to show that, though the main features are explained by this theoretical approach, nevertheless the predicted values both for $u^{2}$ and the solar proton temperature $T_{w}$ are clearly on the low side of the VOYAGER2 data. This may partly be due to mixing of high- and lowvelocity solar wind structures and partly due to the fact that the fraction of adiabatically cooled pick-up ions copopulate the Maxwell tails of the solar wind protons. In view of these open problems, in the following part of the paper we shall reconsider heating sources due to absorption of turbulence energy by solar wind protons.

Smith et al. (2001) describe MHD-waves, both convected and locally generated ones, by one common power spectrum characterized by two parameters, namely the turbulence level and the correlation length. Here, we are going to present below we consider convected background MHD-waves and MHD waves locally generated by pick-up ions as two independent contributions to the turbulence power spectrum resulting in two additive heating sources. We use as a distance-dependent quantity the outer scale $l_{0}=2 \pi / k_{0}$, similar to the correlation length used by Smith et al. (2001), to characterize the evolution of the background turbulence and to describe its contribution to the heating. In our approach here this outer scale increases monotonically with increasing solar distance. The main reason for treating the pick-up ion induced heating source as being separate from the one connected with the background turbulence is due to the fact that the wavenumber $k_{i} \simeq \Omega / V_{w}$ of maximum power generation by pick-up ions (see Huddleston \& Johnstone 1992) is only one order of magnitude smaller than the main dissipation wavenumber $k_{\mathrm{dis}}=\Omega / v_{\mathrm{A}}$ whereas the difference between $k_{i}$ and $k_{0}$ amounts to more than three orders of magnitude.

\subsection{A: Convected turbulence}

It is well known that the solar wind transports turbulent energy distributed over an extended range of wavenumbers $k$, with a typical "flicker" spectrum at low frequencies up to a critical wavenumber $k_{0}$, and with a typical inertial spectrum from the critical wavenumber upwards to the solar proton dissipation wavenumber $k_{\text {dis }} \cong \Omega / v_{\text {A }}$ (e.g. see Goldstein \& Roberts 1999). Here $\Omega$ denotes the proton gyrofrequency, and $v_{\mathrm{A}}$ is the Alfvén velocity. The spectrum of this convected MHD turbulence thus is given by:

$$
W_{k}(k)=D k^{-\eta} \quad \text { for wavenumbers: } k \leqslant k_{0}
$$

and

$$
W_{k}(k)=D k_{0}^{\lambda-\eta} k^{-\lambda} \quad \text { for wavenumbers: } k \geq k_{0}
$$

where $D$ is a structure constant. Here $0 \leq \eta \leq 1$ is the spectral index of the so-called "flicker" spectrum, and $\lambda$ is the spectral index of the inertial spectrum, i.e. $\lambda=5 / 3$ for the Kolmogorov turbulence, or $\lambda=3 / 2$ for the Kraichnan turbulence.

We consider now the locally constant wavepower flux in the inertial branch of the spectrum given by Zhou \& Matthaeus (1990) or, in a more general form, by Tu \& Marsch (1995)

$\Phi_{k}(r)=-D_{k k} \frac{\partial}{\partial k} W_{k}=-\lambda C_{k k} k v_{\mathrm{A}}\left(4 \pi k W_{k} / B^{2}\right)^{\chi}\left(k W_{k}\right)$

where $D_{k k}$ is the nonlinear wave-wave diffusion coefficient for isotropic turbulence, $C_{k k} \approx 0.1=$ const., $B$ is the static background magnetic field, and with $\chi=1 / 2$ (or $\chi=1$ ) for the Kolmogorov turbulence (or for the Kraichnan turbulence), respectively. The flux $\Phi_{k}(r)$ given by Eq. (6) represents a constant in the non-dissipative range. Its value can simply be found by evaluating the above equation at the critical wavenumber $k_{0}$, where the wavepower is given by

$W_{k}\left(k_{0}\right)=D k_{0}^{-\eta}$.

This then leads to the wavepower flux:

$\Phi_{k}\left(k=k_{0}, r\right)=\lambda C_{k k} v_{\mathrm{A}} k_{0}^{1+(\chi+1)(1-\eta)} D^{\chi+1}\left(4 \pi / B^{2}\right)^{\chi}$.

We make use here of the expected radial dependence of the critical wavenumber $k_{0}$ and the average field fluctuation energy. From Chashei \& Shishov (1982) and Chashei (1986) requiring that at $k_{0}$ the rates of linear processes and nonlinear interactions should be equal we then obtain

$V_{w} k_{0} W_{k}\left(k_{0}\right) \approx r \Phi_{k}\left(k_{0}\right)$

where $V_{w}$ is the solar wind speed. Combining Eqs. (6) through (8) one can find the following relation for the turbulence critical scale $k_{0}$,

$k_{0}^{1+\chi(1-\eta)}=\left(V_{w} / C_{k k} r v_{\mathrm{A}}\right)\left(B^{2} / 4 \pi D\right)^{\chi}$.

Taking into account Eq. (9) we can now evaluate $\Phi_{k}\left(k_{0}\right)$ given in Eq. (8):

$\Phi_{k}\left(k_{0}\right)=\left(V_{w} / r\right) D k_{0}^{1-\eta}$.

We furthermore consider the radial dependence of the critical wavenumber $k_{0}(r)$ and of the structure constant $D(r)$. For that purpose we assume, that based on the confirmed validity of Parker's field $B$ (see e.g. Balogh et al. 1992) and a spherically symmetric solar wind expansion geometry at distances $r \geq 1 \mathrm{AU}$, the following relations are valid:

$B^{2}=B_{0}^{2}\left(r_{0} / r\right)^{2}$

and

$v_{\mathrm{A}}=B /\left(4 \pi \rho_{w}\right)^{1 / 2}=v_{\mathrm{A} 0}=$ const.

The structure constant $D$ is proportional to the wavenumber average of the magnetic field fluctuation power, i.e. to 
$<\delta B^{2} / 4 \pi>$, and in the low frequency spectral range is dominated by linear processes, thus

$D(r) / D\left(r_{0}\right)=<\delta B_{\text {lin }}^{2}(r)>/<\delta B^{2}\left(r_{0}\right)>$

where $<\delta B_{\text {lin }}^{2}(r)>=D(r) k_{0}^{1-\eta}\left(r_{0}\right)$. We assume below that

$D(r)=D\left(r_{0}\right)\left(r_{0} / r\right)^{2}$

for distances $r \geq 1 \mathrm{AU}$.

The following arguments can be presented in favour of the radial dependence adopted in Eq. (12):

It is well known that the wave vectors of Alfvén waves have a tendency to approach the radial direction at increasing distances from the sun (Völk \& Alpers 1973; Hollweg 1975). Under these conditions, MHD turbulence convected outwards consists of right-handed and left-handed MHD waves which can be treated (see Malara \& Elaoufir 1991; Grappin et al. 1993) as typical Alfvén wave instabilities with respect to the excitation of magnetosonic waves, i.e. the Alfvén waves propagating at large angles to the quasi-azimuthal ambient magnetic field become unstable with respect to exciting magnetosonic waves. Thus if the relevant magnetic field disturbances are connected with Alfvén waves then their energy density has to fall off as $<\delta B^{2}(r) / 4 \pi>_{\mathrm{A}} \sim r^{-3}$ in the WKB approach at distances $r \geq 1 \mathrm{AU}$, while the radial dependence would be slower for the fast magnetosonic waves, rather falling off as $<\delta B^{2}(r) / 4 \pi>_{\mathrm{F}} \sim r^{-2}$. Indeed, the level or turbulence $\delta B^{2}$ in the WKB approach can be found from the principle of wave action flux conservation given by:

$r^{2} V_{w}\left(\omega / \omega^{\prime}\right) \delta B^{2}=$ const.

where $\omega$ and $\omega^{\prime}$ are the wave frequencies in the immovable rest frame and in the frame co-moving with the solar wind plasma bulk, respectively. In the case of Alfvén waves $\left(\omega / \omega^{\prime}\right)$ is proportional to $r$, since $\omega^{\prime} \sim k_{\|} \sim(1 / r)$, and in case of fast magnetosonic waves $\left(\omega / \omega^{\prime}\right)$ is approximately constant, because $\omega^{\prime} \sim k_{\perp} \simeq$ const., where $k_{\|}$and $k_{\perp}$ are the components of the wave vectors parallel and perpendicular to the ambient magnetic background field.

The radial dependence of the magnetic field fluctuations adopted in Eq. (12) for regions $r \geq 1 \mathrm{AU}$ was also supported by Jokipii \& Kota (1989) and Zank et al. (1996). For these reasons we can expect that the local MHD turbulence at $r \geq 1 \mathrm{AU}$ represents a mixture of Alfvén and magnetosonic waves, with $<\delta B^{2}>_{\mathrm{A}} \approx<\delta B^{2}>_{\mathrm{F}}$ assumed as being valid, but the fast magnetosonic waves finally are responsible for the successive radial transport of turbulent energy at larger distances. In this case the relation (11) is valid, and the radial dependence of $k_{0}(r)$ in Eq. (9) is defined only by the first factor, since the second factor is a constant, i.e. $B^{2} / D=$ const. Assuming futhermore that $<\delta B_{0}^{2} / 4 \pi>\approx G_{0} \rho_{0} v_{\mathrm{A} 0}^{2}$ with $G_{0}=$ const. $\leq 1$ being the fractional turbulence level at $r=r_{0}=1 \mathrm{AU}$, and, consequently, $k_{0}\left(r_{0}\right) \approx V_{w} /\left(v_{\mathrm{A} 0} r_{0}\right)$, we finally obtain:

$\Phi_{k}\left(k=k_{0}, r\right)=\Phi_{0} \times\left(r / r_{0}\right)^{-s}$

with

$\Phi_{0}=\Phi_{k}\left(k=k_{0}, r_{0}\right)=G_{0}\left(V_{w} B_{0}^{2}\right) /\left(4 \pi r_{0}\right)$ and

$s=[3+3(\chi+1)(1-\eta)] /[1+\chi(1-\eta)]$.

In the special cases of Kolmogorov $(\chi=1 / 2)$ turbulence or Kraichnan $(\chi=1)$ turbulence we thus have from Eq. (15):

$s=(11-5 \eta) /(3-\eta)$ at $\chi=1 / 2$,

or

$s=(7-4 \eta) /(2-\eta) \quad$ at $\quad \chi=1$.

In the most interesting case of the low frequency "flicker" spectrum with $\eta=1$ (Matthaeus \& Goldstein 1986; Hourbury \& Balogh 2001) the radial profile of Eq. (13) does not depend on the turbulence model, and in both cases leads to $s=3$. A decrease in $\eta$ up to $\eta=0$ in case of a flat spectrum leads to an increase in $s$ in Eq. (15) up to an extreme value of $s=11 / 3$ in the case of the Kolmogorov turbulence and up to $s=7 / 2$ in the case of the Kraichnan turbulence. In any case, the radial decrease of $\Phi_{k}\left(k=k_{0}, r\right)$ given in Eq. (13) is considerably slower, than that considered by Smith et al. (2001) for purely Alfvénic turbulences when $s>4$.

When estimating the heating source due to convected turbulences we neglected a possible anisotropy in the wavenumber vector distribution of the turbulence power which is unfortunately not known in detail (Goldstein \& Roberts 1999). However, even taking anisotropies into account will not result in considerable changes in our conclusions because we used only rough energetic characteristics of the solar wind turbulence, such as the fractional level of fluctuations and the turbulence outer scale. The turbulent energy $\Phi_{k}\left(k=k_{0}, r\right)$ is locally cascading down to the solar wind proton dissipation scale, i.e. to the wavenumber $k_{\mathrm{dis}}=\Omega / v_{\mathrm{A}}$, and is absorbed by solar wind protons there. Turbulent energy cascading in the $k_{\|}$- wavenumber space is caused by nonlinear wave - wave interactions with the cooperation of Alfvén and magnetosonic waves.

Fractional absorption of $\Phi_{k}$ prior to arriving at wavenumbers $k_{\text {dis }}$ by dissipation to heavier solar wind ions obviously can be also neglected in view of inertial power law spectra which are observed up to the highest frequencies (see Leamon et al. 1998; Goldstein \& Roberts 1999). Convected turbulent energy thus serves as one of two relevant heat sources for distant solar wind protons considered in this paper. The other one is connected with the turbulent energy generated by pick-up ions through their free kinetic energy immediately after injection and, since then partly also cascading to the proton dissipation scale by means of nonlinear wave-wave interactions, also serves as a relevant heat source which we consider next.

\subsection{B: Pick-up ion-generated turbulence}

Pick-up ions are produced by ionization of interstellar neutral atoms in the heliosphere. Then they are convected outwards with the solar wind flow constituting a separate suprathermal ion fluid. The thermodynamic behaviour of this pick-up ion fluid at its motion outwards to the outer heliosphere till now has not been fully understood. Some new aspects, however, of their thermodynamic action were discussed in recent papers by 
Williams et al. (1995), Fahr \& Rucinski (1999, 2001, 2002) and Smith et al. (2001). Pick-up ions could excite waves by virtue of their initial distribution function which is unstable with respect to the excitation of wave power (see e.g. Wu \& Davidson 1972; Hartle \& Wu 1973; Lee \& Ip 1987; Freund \& Wu 1988; Fahr \& Ziemkiewicz 1988; Gray et al. 1996). While immediately after their injection pick-up ions first generate some wavepower via kinetic instabilities they, after some first-order pitch-angle isotropization has occured, also experience Fermi-2 energizations (energy diffusion) by nonlinear wave-particle interaction with already preexisting, convected wave turbulences (see Chalov et al. 1995, 1997; Fichtner et al. 1996; le Roux \& Fichtner 1997).

In the following we consider the balance of these relevant energy production mechanisms and study the effect of the pickup ion-generated turbulent energy at a consecutive absorption by solar wind protons which indicates the possibility that solar wind protons might be globally and continuously heated at their motion to larger solar distances. Since we thereby do not aim at a kinetic description, but simply want to describe the thermodynamics of solar wind protons on the basis of their pressure $P_{w}$, it suffices to take into account the total dissipation of turbulent energy at whatever wavenumber due to pick-up ion induced wave power cascading down to the main dissipation scale, i.e. up to $k_{\mathrm{dis}} \simeq \Omega_{\mathrm{p}} / v_{\mathrm{A}}$.

We assume that pick-up ions just after their injection undergo fast pitch-angle scattering, redistributing them from an initial torus configuration in velocity space onto a bi-spherical hemispheric shell configuration (see Huddleston \& Johnstone 1992). Rigorously taken, even in this bi-spherical mode, pickup ions have not yet attained a strictly pitchangle-isotropic distribution function and still may continue to generate some more turbulent energy which, however, is poorly quantifyable and thus is not taken into account in our following calculation. We instead consider only the free energy of the primary pick-up ions pumped into the turbulent wave field untill they arrive at the quasistatic bi-spherical distribution. We intend to calculate this energy in the following paragraphs.

At larger distances the tilt of the magnetic field $\vec{B}$ (especially in the ecliptic) quickly becomes nearly rectangular with respect to the radial solar wind velocity $\overrightarrow{V_{w}}$. Thus the associated bi-spheres are nearly symmetrically configured around the systems of upgoing and downgoing Alfvén waves and they are equally distributed by newly created pick-up ions. Under these conditions at the event of injection the pick-up ion velocity in the SW frame is equal to the solar wind velocity $V_{w}$. Consequently after pitch angle scattering to the accessible bispheres (i.e. no energy gain!) within each of these fractional shells for upstream and downstream waves one derives the following permitted pick-up ion velocity $v$ when judged in the SW frame by:

$v^{2}=v_{\mathrm{A}}^{2}+\left(V_{w}^{2}+v_{\mathrm{A}}^{2}\right)-2 v_{\mathrm{A}} \sqrt{V_{w}^{2}+v_{\mathrm{A}}^{2}} \cos \vartheta$

where $\vartheta$ is the pitch angle of the resulting velocity $v$ in the upstream wave frame. Since the newly generated pick-up ions are assumed to quickly become randomly distributed on the accessible spherical shells, the distribution function for the populated velocities $v$ is then simply given by the associated velocity space volume $\Delta v=v \mathrm{~d} \cos \vartheta(v)$, i.e. is hence given by:

$$
f(v)=\frac{-\mathrm{d} \cos \vartheta(v)}{1-\cos \vartheta_{\max }}
$$

where the maximum possible tilt angle $\vartheta_{\max }$ is simply given by:

$$
\cos \vartheta_{\max }=\frac{v_{\mathrm{A}}}{\sqrt{V_{w}^{2}+v_{\mathrm{A}}^{2}}}
$$

From the above expression (17) one obtains the mean squared velocity $\left\langle v^{2}(\vartheta)\right\rangle$ of the bi-spherically distributed pick-up ions with the following expression:

$$
\begin{aligned}
& \left\langle v^{2}(\vartheta)\right\rangle=\frac{1}{1-\frac{v_{\mathrm{A}}}{\sqrt{V_{w}^{2}+v_{\mathrm{A}}^{2}}}} . \\
& \int_{\cos \vartheta_{\max }}^{1}\left[v_{\mathrm{A}}^{2}+\left(V_{w}^{2}+v_{\mathrm{A}}^{2}\right)-2 v_{\mathrm{A}} \sqrt{V_{w}^{2}+v_{\mathrm{A}}^{2}} X\right] \mathrm{d} X
\end{aligned}
$$

which easily becomes:

$$
\left\langle v^{2}(\vartheta)\right\rangle=V_{w}^{2}\left[1-\frac{v_{\mathrm{A}}}{\sqrt{V_{w}^{2}+v_{\mathrm{A}}^{2}}}\right]+2 v_{\mathrm{A}}^{2}
$$

and now allows one to respect the fact that the energy $\Xi_{i}=$ $\frac{1}{2} m_{\mathrm{p}} V_{w}^{2}$ of primarily injected pick-up ions after a first violent period of wave-driving is reduced to the average energy $\epsilon_{i}=\frac{1}{2} m_{\mathrm{p}}\left\langle v^{2}(\vartheta)\right\rangle$. This means that in average the energy $\Delta \epsilon_{i}=$ $\Xi_{i}-\epsilon_{i}$ is pumped into the turbulent wave field, mainly occuring at or around the injection wavenumber $k_{i} \simeq \Omega_{\mathrm{p}} / V_{w}$ (see e.g. Huddleston \& Johnstone 1992). The loss of free energy to the wave fields with the change of the redistribution from the initial torus configuration into the bi-spherical configuration is thereby properly taken into account.

Now we assume that this energy input $\Delta \epsilon_{i}$ into the turbulent wavepower is producing a local power peak at $k_{i}$ with decreasing spectral power left and right of the injection wavenumber due to wave power diffusion. It can thus be assumed that due to nonlinear wave-wave interactions, i.e. diffusion in $k$-space, this energy input is cascading up and down from $k_{i}$, more or less at equal parts, i.e. one half of it cascades to smaller wavenumbers and thus may contribute to Fermi- 2 accelerations of pickup ions to higher energies, the other half cascades up to larger wavenumbers and finally is absorbed by solar wind protons at the dissipation wavenumber $k_{\mathrm{dis}}=\Omega_{\mathrm{p}} / v_{\mathrm{A}}$.

The energy injection to solar wind protons due to pick-up ion wave energy thus is given by:

$$
Q_{i}(r)=\beta_{\mathrm{ex}}(r) \Delta \epsilon_{i} \frac{1}{4}=\frac{1}{4} \beta_{\mathrm{ex}}(r) m_{\mathrm{p}}\left(V_{w}^{2} \frac{v_{\mathrm{A}}}{\sqrt{V_{w}^{2}+v_{\mathrm{A}}^{2}}}-2 v_{\mathrm{A}}^{2}\right) .
$$

Here $\beta_{\text {ex }}(r)$ denotes the local charge exchange rate of solar wind protons and interstellar $\mathrm{H}$-atoms also describing the local rate of pick-up proton injections. This rate is given by:

$\beta_{\mathrm{ex}}(r)=n_{w}(r) n_{\mathrm{H}}(r) \sigma_{\mathrm{ex}} V_{w}$ 
where $n_{w}$ and $n_{\mathrm{H}}$ are the local proton and $\mathrm{H}$-atom densities, respectively, $\sigma_{\text {ex }}$ is the charge exchange cross section and $V_{w}$ is the solar wind velocity.

For the upwind hemisphere the following approximative, so-called "cold" representation of the H-atom density can be used here (see Fahr 1971; Axford 1972):

$n_{\mathrm{H}}(r, \Theta)=n_{\mathrm{H} \infty} \exp \left(-\frac{\beta_{0} r_{0}^{2} \Theta}{V_{\mathrm{H} \infty} r \sin \Theta}\right)$

where $\Theta$ denotes the off-wind angle, $\beta_{0} \simeq n_{w 0} \sigma_{\mathrm{ex}} V_{w}$ is the ionization rate of $\mathrm{H}$-atoms at the reference distance $r_{0}$, and where $V_{\mathrm{H} \infty}$ is the bulk velocity of the inflowing interstellar H-atoms at large distances.

To apply formula (23) to the upwind axis one simply considers this formula in the limit $\Theta \rightarrow 0$ and then obtains:

$n_{\mathrm{H}}\left(r, \Theta=0^{\circ}\right)=n_{\mathrm{H} \infty} \exp \left(-\frac{n_{w 0} \sigma_{\mathrm{ex}} V_{w} r_{0}^{2}}{V_{\mathrm{H} \infty} r}\right)$.

For the downwind axis, i.e. for $\Theta=\pi$, one has, however, to take into account the interstellar $\mathrm{H}$-atom temperature $T_{\mathrm{H}, \infty}$ and here, when solar gravity is compensated by solar H-Lyman-Alpha radiation pressure, one can use the following approximative representation:

$n_{\mathrm{H}}(r, \Theta=\pi)=n_{\mathrm{H} \infty} \exp \left(-\frac{\beta_{0} r_{0}^{2} \Theta^{t}}{V_{\mathrm{H} \infty} r \sin \Theta^{t}}\right)$

with the angle $\Theta^{t}$ given by:

$\Theta^{t}=\pi-\operatorname{arctg}\left(\sqrt{\frac{2 K T_{\mathrm{H} \infty}}{m_{\mathrm{p}} V_{\mathrm{H} \infty}^{2}}}\right)$.

Coming back to the Eq. (21) one can simplify the expression for $Q_{i}$ by setting:

$Q_{i}(r)=\frac{1}{4} \beta_{\mathrm{ex}}(r) m_{\mathrm{p}} V_{w}^{2} \epsilon_{\mathrm{f}}$.

Here the following shorthand notation for the factor $\epsilon_{\mathrm{f}}$ has been introduced:

$\epsilon_{\mathrm{f}}=\frac{\mu}{\sqrt{1+\mu^{2}}}-2 \mu^{2} \simeq \mu-2 \mu^{2}-\frac{1}{2} \mu^{3} \simeq \mu_{0}\left(1-2 \mu_{0}\right)$

where $\mu=v_{\mathrm{A}} / V_{w} \simeq v_{\mathrm{A} 0} / V_{w 0} \ll 1$ was used. It should be noted that in the other limiting case, when the dissipated energy is distributed between the primary protons and pick-up ions proportionally to their abundances, the factor $1 / 4$ in Eq. (27) should be replaced with the value approximately equal to $1 / 2$.

\section{Thermodynamics of wave-heated solar wind protons}

We now give the equation for the thermodynamic behaviour of the solar wind protons in terms of proton pressure $P_{w}$ under the effects of adiabatic cooling and of heating both by convected turbulence energy $\Phi_{k}\left(k=k_{0}, r\right)$ and by locally originating turbulent energy $Q_{i}$ pumped into the wavefield by freshly injected pick-up ions. For the proton thermal pressure $P_{w}$ we then obtain the following equation (see Fahr \& Ziemkiewicz 1988; or Fahr \& Rucinski 2002):

$$
\begin{array}{r}
\operatorname{div}\left(\frac{\gamma}{\gamma-1} P_{w} \overrightarrow{V_{w}}\right)-\left(\overrightarrow{V_{w}} \cdot \operatorname{grad}\right) P_{w}= \\
-\beta_{\mathrm{ex}}\left(K T_{w}\right)+Q_{b}(r)+Q_{i}(r)
\end{array}
$$

where

$Q_{b}(r)=\Phi_{k}\left(k=k_{0}, r\right)$

and

$Q_{i}(r)=\frac{1}{4} \beta_{\text {ex }}(r) m_{\mathrm{p}} V_{w}^{2} \mu_{0}\left(1-2 \mu_{0}\right)$.

Furthermore $K$ is the Boltzmann constant, and $m_{\mathrm{p}}$ is the proton mass. The left-hand side of the above equation describes the divergence of the proton enthalpy flow and the work done by the pressure $P_{w}$, while the right-hand side represents energy sinks due to the charge-exchange losses of thermal proton energy $K T_{w}$ and energy sources due to absorption of convected turbulent energy $Q_{b}(r)$ (defined by Eqs. (13) and (14) and of turbulent energy pumped by pick-up ions $Q_{i}(r)$ (defined by Eq. (27)).

Below we will make use of the assumption $V_{w}=V_{w 0} \gg$ $v_{\mathrm{A}}=v_{\mathrm{A} 0}$ valid at distances $r \geq 1 \mathrm{AU}$. We also assume that the solar wind plasma can be considered as a one-atomic ion gas, suggesting $\gamma=5 / 3$. Furthermore, comparing the chargeexchange energy sink and the pick-up ion induced source on the right-hand side of Eq. (28), one can find that

$$
\begin{aligned}
& \beta_{\mathrm{ex}}\left(K T_{w}\right) / Q_{i}(r)=\left(4 K T_{w}\right) /\left(m_{\mathrm{p}} V_{w} V v_{\mathrm{A}}\right)= \\
& 2 c_{w}^{2} /\left(V_{w} v_{\mathrm{A}}\right) \ll 1 \quad \text { for } r \geq 1
\end{aligned}
$$

where $c_{w}$ is the mean thermal speed of solar wind protons. The above inequality shows that the charge-exchange sink can be neglected in the energy Eq. (28).

This equation, if reduced to an equation for the proton temperature $T_{w}$ by setting $P_{w}=n_{w} K T_{w}$, and adopting that $V_{w}=$ const. and $n_{w}=n_{w 0}\left(r_{0} / r\right)^{2}$ can be used, then attains the following form:

$\frac{\mathrm{d} T_{w}}{\mathrm{~d} r}+\frac{4}{3} \frac{T_{w}}{r}=q_{b}(r)+q_{i}(r)$

where as new denotations $q_{b, i}(r)=2 Q_{b, i}(r) / 3 n_{w} K V_{w}$ have been introduced which on the basis of the aforementioned definitions attain the following form:

$q_{b}(r)=\left(2 G_{0} m_{\mathrm{p}} v_{\AA}^{2} / 3 K r_{0}\right)\left(r_{0} / r\right)^{s-2}$

and

$q_{i}(r)=\left(\Lambda m_{\mathrm{p}} V_{w} v_{\mathrm{A}} / 6 K r_{0}\right) \exp \left(-\Lambda \frac{V_{w}}{V_{\mathrm{H} \infty}} \frac{n_{w 0}}{n_{\mathrm{H} \infty}} \frac{r_{0}}{r}\right)$.

Defining $\Lambda=n_{\mathrm{H} \infty} \sigma_{\mathrm{ex}} r_{0}, g_{0}=\Lambda V_{w} / 4 v_{\mathrm{A}}, x=\left(r / r_{0}\right), T_{\mathrm{S}}=$ $2 m_{\mathrm{p}} v_{\mathrm{A}}^{2} / 3 K$ we then find with Eqs. (30) and (31):

$\frac{\mathrm{d} T_{w}}{\mathrm{~d} x}+\frac{4}{3} \frac{T_{w}}{x}=T_{\mathrm{S}}\left[G_{0} x^{2-s}+g_{0} \exp \left(-\Lambda_{1} / x\right)\right]$ 
where $\Lambda_{1}=\Lambda\left(n_{w 0} V_{w} / n_{\mathrm{H} \infty} V_{\mathrm{H} \infty}\right)$. The radial profile of the proton temperature $T_{w}(x)$ at $x \geq 1$ is thus defined by the solution of Eq. (32) given in the following form:

$T_{w}(x)=x^{-4 / 3}\left[T_{w 0}+T_{\mathrm{S}}\left(I_{b}(x)+I_{i}(x)\right)\right]$

where $T_{w 0}$ is the solar wind proton temperature at $x=1$, and the expressions $I_{b}(x)$ and $I_{i}(x)$ are defined by:

$I_{b}(x)=\frac{3 G_{0}}{13-3 s}\left(x^{(13-3 s) / 3}-1\right)$

and

$I_{i}(x)=g_{0} \int_{1}^{x} \varsigma^{4 / 3} \exp \left(-\Lambda_{1} / \varsigma\right) \mathrm{d} \varsigma$.

Instead of by the temperature profile, the heating process can also be characterized by the local effective polytropic index $\gamma_{\mathrm{eff}}=\gamma_{\mathrm{eff}}(x)$ which is defined by

$\gamma_{\mathrm{eff}}=1-\left(\frac{x}{2 T_{w}}\right) \frac{\mathrm{d} T_{w}}{\mathrm{~d} x}=1-\frac{1}{2} \frac{\mathrm{d} \ln T_{w}}{\mathrm{~d} \ln x}$.

Using Eq. (32) and its solution given by Eq. (33) we find the following relation describing the $x$-dependence of $\gamma_{\mathrm{eff}}(x)$ :

$\gamma_{\mathrm{eff}}=5 / 3-T_{\mathrm{S}} \frac{\left[G_{0} x^{(13-3 s) / 3}+g_{0} x^{7 / 3} \exp \left(-\Lambda_{1} / x\right)\right]}{\left[2 T_{w 0}+2 T_{\mathrm{S}}\left(I_{b}(x)+I_{i}(x)\right)\right]}$.

If we neglect any form of heating considered here, i.e. setting $G_{0}=g_{0}=0$, then, evidently, the adiabatic constant is retained with $\gamma_{\mathrm{eff}, 0}=5 / 3$. In the case when the proton temperature is dominated by the absorption of the background turbulence, i.e. if $I_{b}(x) \gg I_{i}(x)$, and $T_{\mathrm{S}} I_{b}(x) \gg T_{w 0}$, then we easily obtain from Eq. (37)

$\gamma_{\mathrm{eff}}=(s-1) / 2$.

The relation (38) shows that $1 \leq \gamma_{\mathrm{eff}} \leq 4 / 3$, because we obtain $9 / 3 \leq s \leq 11 / 3$ for the model of convected turbulence considered here in this paper. It should be noted that only in the case of a low frequency flicker spectrum with a low frequency exponent $\eta=1$ and $s=3$ the proton heating dominated by absorption of convected turbulences would result in an almost isothermal behaviour with $\gamma_{\mathrm{eff}}=1$ and an asymptotic temperature $T_{w, b}(x \rightarrow \infty)=G_{0}\left(m_{\mathrm{p}} v_{\mathrm{A}}^{2} / 2 K\right)$ which can be called the temperature of the MHD waves. This shows the tendency of the approach towards an energy equipartition between MHD waves and solar wind protons with a temperature $T_{w, b}$ which is fully controlled by the background turbulence. In all other cases the temperature $T_{w}$ would decrease with increasing heliocentric distance $r$.

On the other hand, if the solar wind proton heating would be dominated by the pick-up ion source, i.e. if $I_{b}(x) \ll I_{i}(x)$, $T_{\mathrm{S}} I_{i}(x) \gg T_{w 0}$, then we find from Eq. (37):

$\gamma_{\mathrm{eff}}=5 / 3-\frac{x^{7 / 3} \exp \left(-\Lambda_{1} / x\right)}{2 \int_{1}^{x} \varsigma^{4 / 3} \exp \left(-\Lambda_{1} / \varsigma\right) \mathrm{d} \varsigma}$.

We can see from Eq. (39) that for $\Lambda_{1} / x \rightarrow 0$ (pick-up ion)induced heating can even result in a polytropic behaviour with $\gamma_{\mathrm{eff}, i}<1$, i.e. in an increase of the proton temperature $T_{w}(x)$ with increasing heliocentric distance $x$. One can see from Eq. (39) that formally the asymptotic value for $\gamma_{\mathrm{eff}}(x \rightarrow \infty)$ leads to $\gamma_{\mathrm{eff}}(\infty)=1 / 2$. It can be concluded that without (pickup ion)-induced heating, the polytropic index $\gamma_{\mathrm{eff}}$ as given by the more general formula (37) never would fall below 1 .

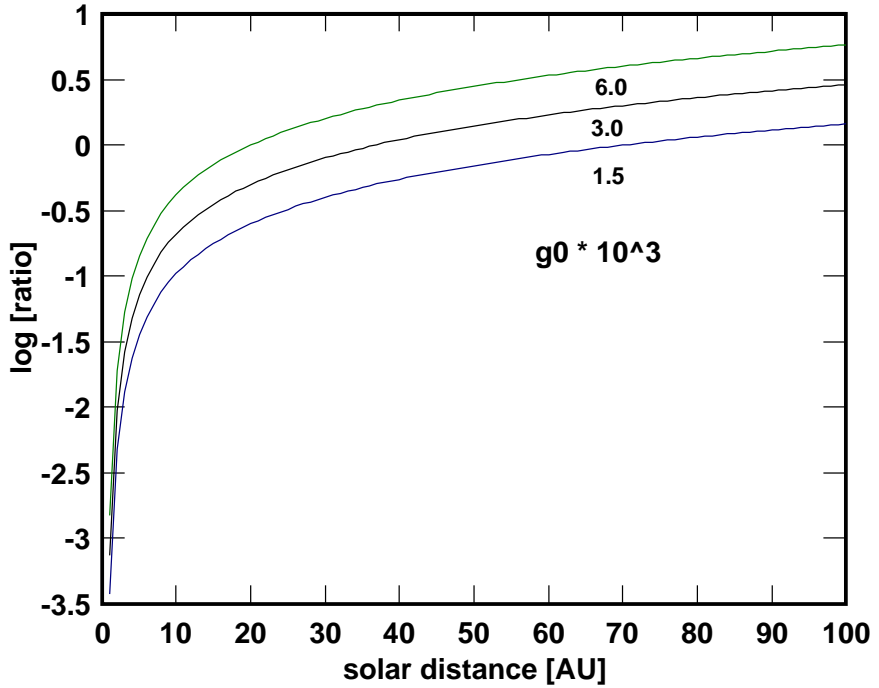

Fig. 1. The ratio $R_{i b}=q_{i} / q_{b}$ is shown as function of the solar distance in units of AU for various values of $g_{0}=1.5 ; 3.0 ; 6,0 \times\left(10^{-3}\right)$. The other relevant parameters are taken to be $G_{0}=0.1$ and $s=9 / 3$.

\section{Concluding remarks}

In the following we shall discuss the most important results of the theoretical derivations presented in the aforegoing sections. We first select appropriate values for the relevant parameters occuring in the calculations. With $n_{\mathrm{H} \infty}=0.05 \mathrm{~cm}^{-3}$ (see e.g. Izmodenov et al. 1999; Izmodenov 2000; Fahr 2000) and $\sigma_{\mathrm{ex}}\left(440 \mathrm{~km} \mathrm{~s}^{-1}\right)=2 \times 10^{-15} \mathrm{~cm}^{2}$ (see e.g. McNutt et al. 1999) one obtains for the standard value of $\Lambda=n_{\mathrm{H} \infty} \sigma_{\mathrm{ex}} r_{0}=$ $1.5 \times 10^{-3}$. With $n_{w 0}=7 \mathrm{~cm}^{-3}$ and $V_{w}=440 \mathrm{~km} \mathrm{~s}^{-1}$ (see Whang 1998) one then obtains the critical ionization distance by $\Lambda_{1} \cdot r_{0}=\Lambda \cdot\left(n_{w 0} V_{w} / n_{\mathrm{H} \infty} V_{\mathrm{H} \infty}\right) \cdot r_{0}=3.7 \mathrm{AU}$. The standard value of the parameter $g_{0}=\Lambda \cdot\left(V_{w} / 4 v_{\mathrm{A}}\right)$ for these standard values thus amounts to $g_{0}=3.3 \times 10^{-3}$ adopting the Alfvén speed with $v_{\mathrm{A}}=50 \mathrm{~km} \mathrm{~s}^{-1}$. As the standard value for $G_{0}=\left\langle\delta B^{2} / 4 \pi\right\rangle /\left(\rho_{0} v_{\mathrm{A}}^{2}\right)$ we may take here $G_{0}=0.1$.

First we want to compare the strength of the local energy sources $q_{b}(r)$ due to absorption of convected turbulence energy and $q_{i}(r)$ due to absorption pumped by pick-up ions turbulence energy. In Fig. 1 we thus display the ratio $R_{i b}(r)$ of the two relevant sources $q_{i}(r)$ and $q_{b}(r)$ given by Eqs. (30) and (31), respectively, obtained in the form:

$R_{i b}(x)=\frac{q_{i}(x)}{q_{b}(x)}=\frac{g_{0}}{G_{0}} \frac{\exp \left(-\frac{\Lambda_{1}}{x}\right)}{x^{2-s}}$.

We can see that dependent on the value adopted for $g_{0}$ essentially determined by the interstellar $\mathrm{H}$-atom density $n_{\mathrm{H} \infty}$, the region of pick-up ion dominance is shifted to regions progressively farther away from the sun with a decrease of $n_{\mathrm{H} \infty}$. For instance an increase of the density $n_{\mathrm{H} \infty}$ from $0.025 \mathrm{~cm}^{-3}$ to $0.01 \mathrm{~cm}^{-3}$ (i.e. $1.5 \times 10^{-3} \leq g_{0} \leq 6 \times 10^{-3}$ ) shifts the region of pick-up ion dominance inwards from $60 \mathrm{AU}$ to $20 \mathrm{AU}$. This clearly manifests that for values of $n_{\mathrm{H} \infty}$ presently under discussion the solar wind proton heating at distances beyond distances of $20 \mathrm{AU}$ always is dominated by turbulence energies pumped by pick-up ions. 


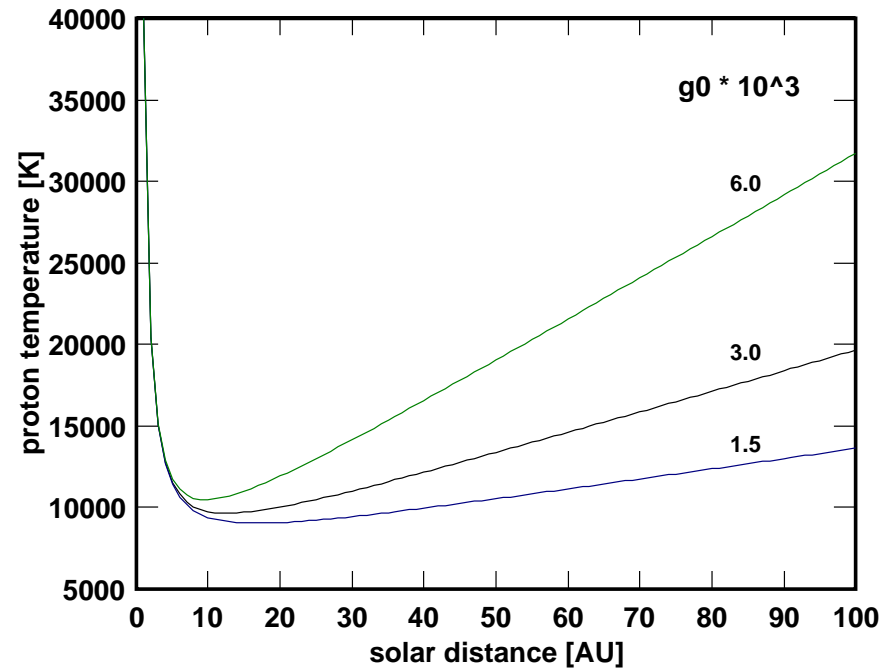

Fig. 2. The temperature $T_{w}$ is shown as function of the solar distance in AU for various values of the parameter $g_{0}$, with $G_{0}=0.1$ and $s=9 / 3$.

The temperature profiles $T_{w}(x)$ resulting with Eq. (33) for these parameter conditions are shown in Fig. 2. As one can see the temperatures $T_{w}(x)$ fall off less steaply, or even increase more strongly with distance $x$, the higher the value $g_{0}$, i.e. the $\mathrm{H}$-atom density $n_{\mathrm{H} \infty}$. Both the temperature itself and the temperature gradient in the outer heliosphere are systematically increased with the increase of $n_{\mathrm{H} \infty}$. How the temperature increases in the outer heliosphere not only is determined by $n_{\mathrm{H} \infty}$, but is also influenced by the properties of the convected turbulence which we have parametrized with the two parameters $G_{0}$ and $s$. As shown in Fig. 3, increasing the relative strength of the convected turbulences from the standard value $G_{0}=0.1$ to values $G_{0}=0.2$ and $G_{0}=0.3$ shifts the location of the temperature minimum outwards from $15 \mathrm{AU}$ to $35 \mathrm{AU}$ and also increases the value of the minimum temperature. On the other hand, as shown in Fig. 4, the increase of the spectral power index $s$ from its standard value $s=9 / 3$ to values $s=10 / 3$ and $s=11 / 3$, though moving the location of the temperature minimum outwards, decreases the value of the temperature minimum.

The variation of the temperature with distance can also be studied in terms of a locally resulting effective polytropic index $\gamma_{\text {eff }}(x)$ derived by Eq. (37). This is done in Fig. 5 adopting our standard parameter values describing the convected turbulences and varying $g_{0}$ (i.e. $n_{\mathrm{Ho}}$ ) in the range between $1.5 \times 10^{-3} \leq g_{0} \leq 6.0 \times 10^{-3}$. As one can notice the index $\gamma_{\mathrm{eff}}$ drops down even to values below 1 with absolute values being lower the higher the value of the H-atom density $n_{\mathrm{H} \infty}$. Two things emerge from this figure: the solar wind protons even not at small distances $1.0 \leq x \leq 5$ behave like an adiabatic fluid but reflect the heating due to absorption of convected turbulences, and second at larger distances in all cases shown, the polytropic index falls below 1, reflecting a temperature increase with the expansion of the solar wind to larger distances. This latter result differs from a similar study carried out by Fahr (2002) where only a drop-off of the effective polytropic index $\gamma_{\mathrm{eff}}(x)$ with distance $x$ to asymptotic values of $\gamma_{\mathrm{eff}}(x \rightarrow \infty) \simeq 1.1 \geq 1$ was obtained. This study by Fahr (2002) was, however, carried

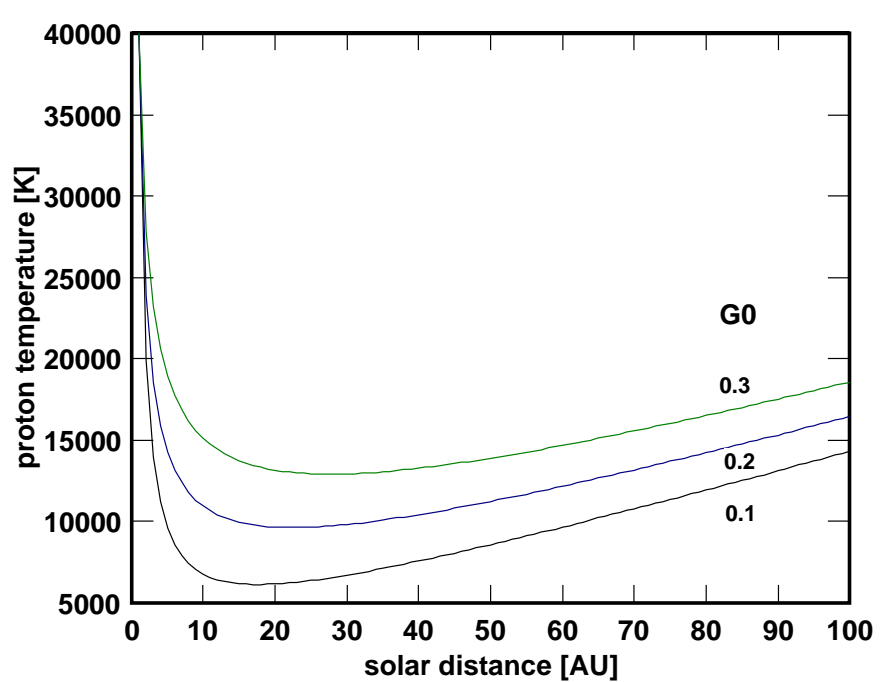

Fig. 3. The temperature $T_{w}$ is shown as function of the solar distance in $\mathrm{AU}$ for various values of the parameter $G_{0}$, with $g_{0}=3 \times 10^{-3}$ and $s=10 / 3$.

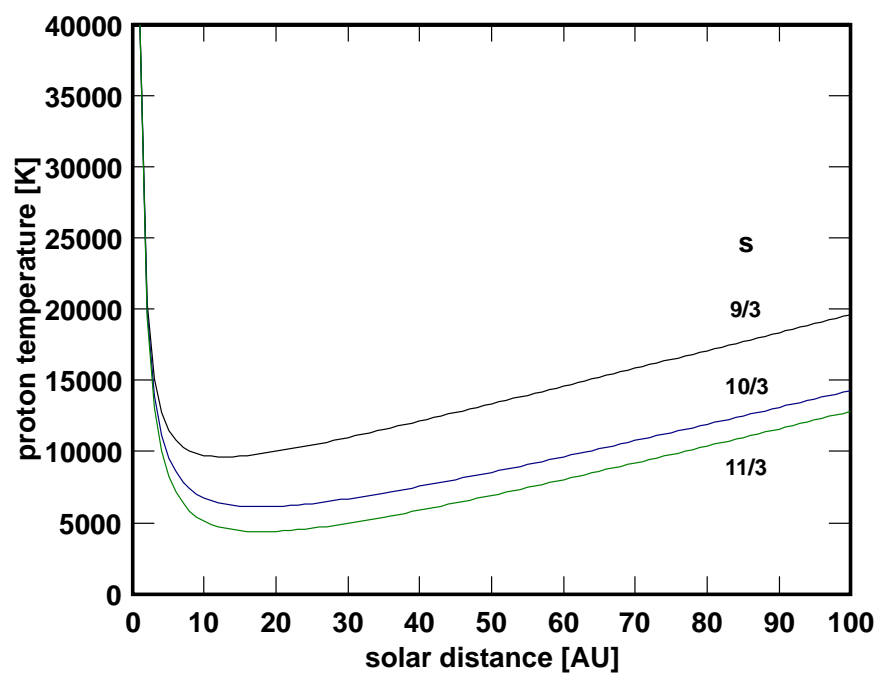

Fig. 4. The temperature $T_{w}$ is shown as function of the solar distance in $\mathrm{AU}$ for various values of the parameter $s$, with $g_{0}=3 \times 10^{-3}$ and $G_{0}=0.1$.

out assuming two important physical prerequisites; first it was asssumed that pick-up ions, when being convected outwards with the solar wind, behave isothermal, and second that the two-fluid solar wind plasma, consisting of original solar wind protons and pick-up ions, as its joint energy source only has available the kinetic energy of freshly injected pick-ups. Here instead we have taken into account that solar wind protons not only profit from the pick-up ion induced heat source, but also from convected turbulence energy absorbed by them. From VOYAGER-1/2 and PIONEER-11 data no clear indication of a temperature increase with distance in the upwind hemisphere can be gained, but it nevertheless seems to be a well established fact that proton temperatures hardly do fall off, if at all, in the region between 20 to $40 \mathrm{AU}$ but rather stay constant there (see Gazis et al. 1994). This nicely seems to be represented by the curves shown in Fig. 3 where it is evident that the level of the constant temperature is variable, perhaps depending on the 


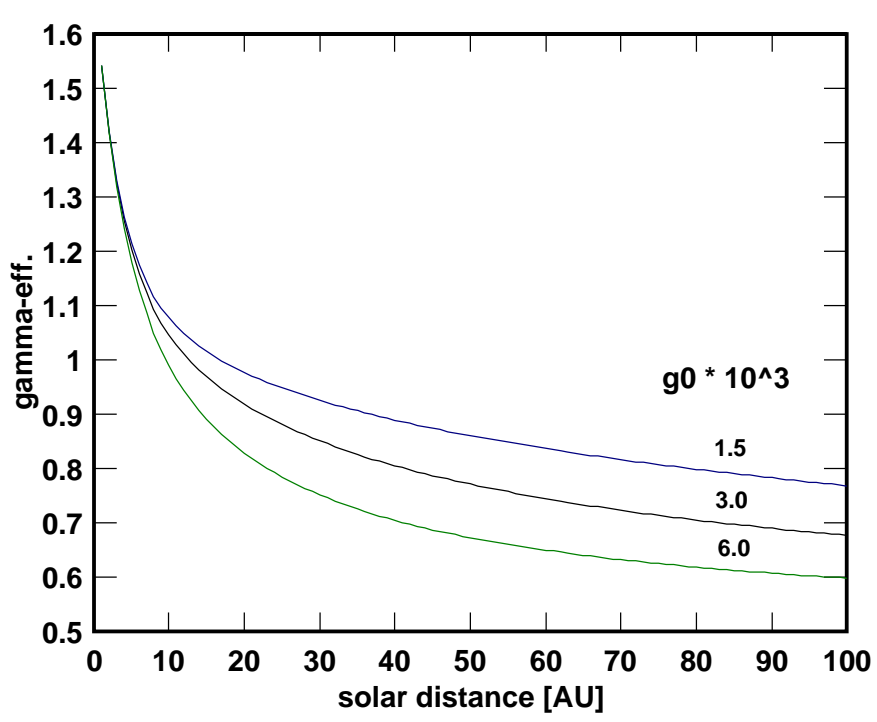

Fig. 5. The polytropic index $\gamma_{\text {eff }}$ is shown as a function of the solar distance in units of AU for various values of the parameter $g_{0}$, with $G_{0}=0.1$ and $s=9 / 3$.

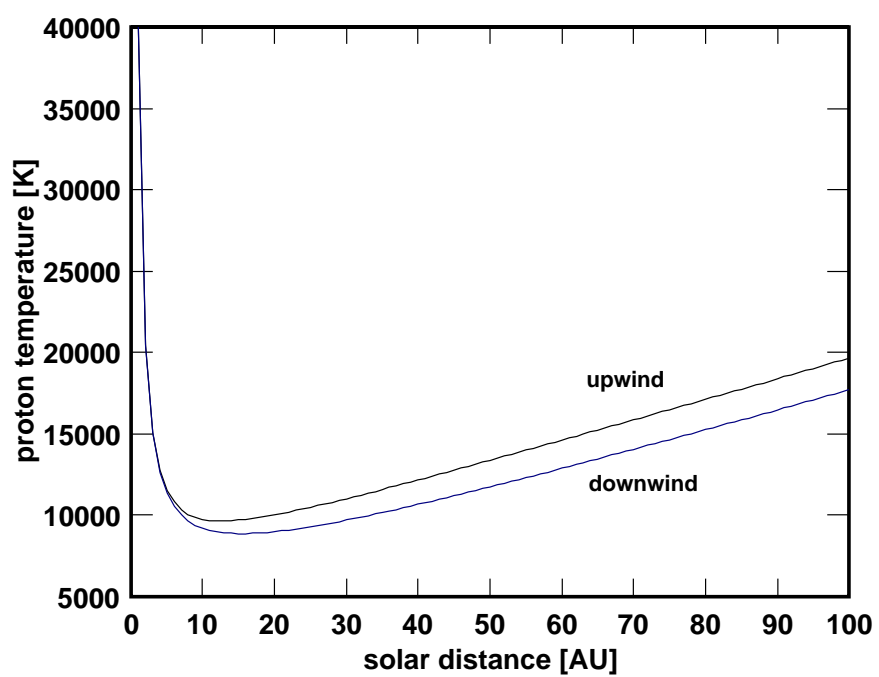

Fig. 6. The temperatures $T_{w}$ in upwind and in downwind direction, respectively, are shown as functions of the solar distance in units of $\mathrm{AU}$ for the parameters $g_{0}=3 \times 10^{-3}, G_{0}=0.1$ and $s=9 / 3$.

level of turbulence parametrized by $G_{0}$. The theoretical values of $T_{w}$ for heliocentric distances larger than $10 \mathrm{AU}$ given in Fig. 3 also are in reasonably good agreement with corresponding values found by Gazis et al. (1994) with VOYAGER-1/2 and PIONEER-11.

Furthermore, it is very interesting to study the temperature profile on the downwind side of the heliosphere. For that purpose in Figs. 6 and 7 we compare temperature results obtained with Eq. (23) for the upwind axis with those obtained with Eq. (25) for the downwind axis. As one can notice the pick-up ion induced heating of the solar wind protons is less pronounced on the downwind side, resulting in lower temperatures and weaker temperature increases there. This result is evidently connected with $\mathrm{H}$-atom depletions and thus lower pick-up ion injection rates on the downwind side (see e.g. Fahr \& Rucinski 1999) and seems to be supported by data

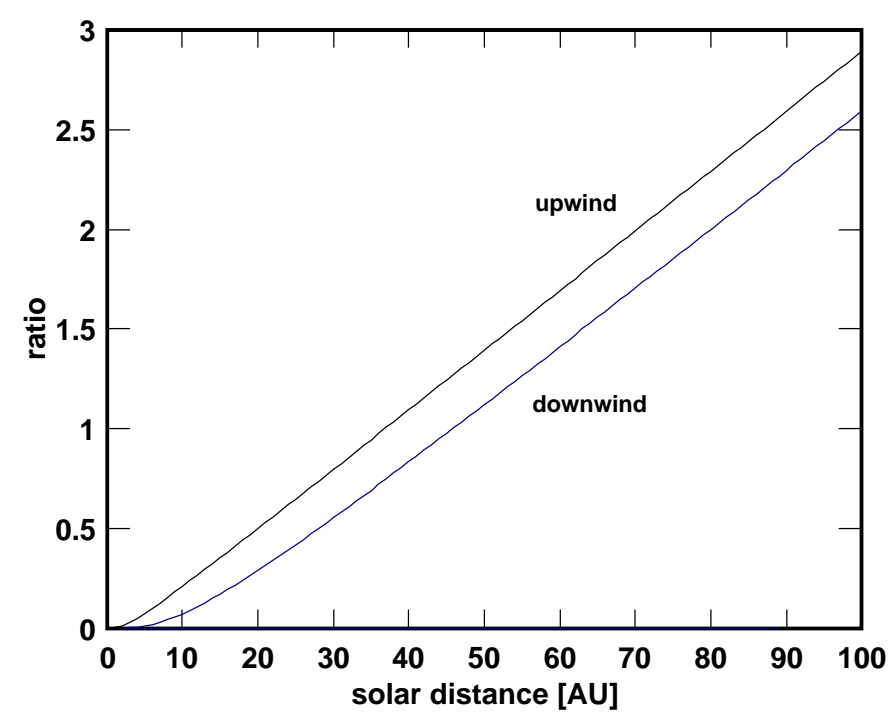

Fig. 7. The ratios $R_{i b}=q_{i} / q_{b}$ in upwind and in downwind direction, respectively, are shown as functions of the solar distance in units of AU for the parameters $g_{0}=3 \times 10^{-3}, G_{0}=0.1$ and $s=9 / 3$.

obtained with PIONEER-11 observations on the upwind, and PIONEER-10 observations on the downwind side, since Gazis et al. (1994) emphasize the fact that PIONEER-11 temperatures are significantly higher than PIONEER-10 temperatures, suggesting the existence of a temperature gradient with solar heliographic longitude, at least in the period of the solar activity minimum. It should be noted that the difference between upwind and downwind temperatures given in Fig. 6 is not very strong. A very careful analysis of observational data is needed to disentangle this difference in measured upwind/downwind proton temperature data from differences caused by different conditions of convected MHD turbulence during the solar activity cycle.

Acknowledgements. This work was partially funded by a NATO Scientific Research Grant (CLG number: 69-5109) and by a binational cooperation project with number 00-02-04022 jointly sponsored by the Deutsche Forschungsgemeinschaft (DFG) and the Russian Foundation of Basic Research (RFBR).

\section{References}

Axford, W. I. 1973, in Solar Wind V, NASA SP-308, 609

Balogh, A., Beek, T. J., Forsyth, R. J., et al. 1992, A\&AS, 92, 221

Chalov, S. V., Fahr, H. J., \& Izmodenov, V. 1995, A\&A, 304, 609

Chalov, S. V., Fahr, H. J., \& Izmodenov, V. 1997, A\&A, 320, 659

Chashei, I. V. 1986, Geomagnetizm and Aeron., 26, 486

Chashei, I. V., \& Shishov, V. I. 1982, Geomagnetizm and Aeron., 22, 529

Coleman, P. 1968, ApJ, 153, 371

Cranmer, S. R. 2001, J. Geophys. Res., 106, 24937

Fahr, H. J. 1971, A\&A, 14, 263

Fahr, H. J. 1973, Sol. Phys., 30, 193

Fahr, H. J. 2000, Astrophys. Space Sci., 274, 35

Fahr, H. J. 2002, Nonlinear Processes in Geophysics, 9, 377

Fahr, H. J., \& Fichtner, H. 1995, Sol. Phys., 158, 353

Fahr, H. J., \& Lay, G. 2000, A\&A, 356, 327

Fahr, H. J., \& Ripken, H. W. 1984, A\&A, 139, 551

Fahr, H. J., \& Rucinski, D. 1999, A\&A, 350, 1071 
Fahr, H. J., \& Rucinski, D. 2001, Space Sci. Rev., 97, 407

Fahr, H. J., \& Rucinski, D. 2002, Nonlinear Processes in Geophys., 9, 377

Fahr, H. J., \& Ziemkiewicz, J. 1988, A\&A, 202, 295

Fichtner, H., le Roux, J. A., Mall, U., \& Rucinski, D. 1996, A\&A, 314, 650

Freund, H. P., \& Wu, C. S. 1988, J. Geophys. Res., 93, 14277

Gazis, P. R., Barnes, A., Mihalov, J. D., \& Lazarus, A. J. 1994, J. Geophys. Res., 99, 6561

Goldstein, M. L., \& Roberts, D. A. 1999, Physics of Plasmas, 6(11), 4155

Grappin, R., Velli, M., \& Mangeney, A. 1993, Phys. Rev. Lett., 70, 2190

Gray, P. C., Smith, C. W., Matthaeus, W. H., \& Otani, N. F. 1996, Geophys. Res. Lett., 23, 113

Hartle, R. E., \& Wu, C. S. 1973, J. Geophys. Res., 78, 5802

Hollweg, J. V. 1975, Rev. Geophys. Space Phys., 13, 263

Holzer, T. E., \& Leer, E. 1973, Ap\&SS, 24, 335

Hourbury, T. S., \& Balogh, A. J. 2001, J. Geophys. Res., 106. 15929

Huddleston, D. E., \& Johnstone, A. D. 1992, J. Geophys. Res., 97, 12217

Izmodenov, V. V. 2000, Ap\&SS, 274, 55

Izmodenov, V. V., Geiss, J., Lallement, R., et al. 1999, J. Geophys. Res, 104, 4731

Jokipii, J. R., \& Kota, J. 1989, Geophys. Res. Lett., 16, 1

Leamon, R. J., Smith, C. W., Ness, N. F., Matthaeus, W. H., \& Wong, H. K. 1998, J. Geophys. Res., 103, 4775

Lee, M. A. 1997, in Effects of cosmic rays and interstellar gas on the dynamics of a wind, ed. J. R. Jokopii, C. P. Sonett, \& M. Giampapa (Stellar Winds, Tucson, Arizona), University of Arizona, 857
Lee, M. A., \& Ip, W. H. 1987, J. Geophys. Res., 92, 11041

Le Roux, J. A., \& Fichtner, H. 1997, J. Geophys. Res., 102, 17365

Liewer, P. C., Velli, M., \& Goldstein, B. E. 2001, J. Geophys. Res., 106, 29261

Malara, F., \& Elaoufir, J. 1991, J. Geophys. Res., 96, 7641

Marsch, E., Goertz, C. K., \& Richter, K. 1982, J. Geophys. Res., 87, 5030

Matthaeus, W. H., \& Goldstein, M. L. 1986, Phys. Rev. Lett., 57, 495

Mattheaus, W., Oughton, D., Pontius, D., \& Zhou, Y. 1994, J. Geophys. Res., 99, 19267

McNutt, R. L., Lyon, J., \& Goodrich, C. C. 1999, J. Geophys. Res., 104,14803

Parker, E. N. 1964, ApJ, 139, 93

Richardson, J. D., Paularena, K. I., Lazarus, A. J., \& Belcher, J. W. 1995, Geophys. Res. Lett., 22, 325

Ripken, H. W., \& Fahr, H. J. 1983, A\&A, 122, 181

Smith, C. V., Matthaeus, W. H., Zank, G. P., et al. 2001, J. Geophys. Res., 106, 8523

Tu, C. Y., \& Marsch, E. 1995, Space Sci. Rev., 73, 1

Vasyliunas, V. M., \& Siscoe, G. L. 1976, J. Geophys. Res., 81, 1247

Völk, H. J., \& Alpers, W. 1973, Astrophys. Space Sci., 20, 267

Whang, Y. C. 1998, J. Geophys. Res., 103, 17419

Whang, Y. C., Lu, J. Y., \& Burlaga, L. F. 1999, J. Geophys. Res., 104, 28255

Williams, L. L., Zank, G. P., \& Matthaeus, W. H. 1995, J. Geophys. Res., 100, 17059

Wu, C. S., \& Davidson, R. C. 1972, J. Geophys. Res., 77, 5399

Wu, F. M., \& Judge, D. L. 1979, ApJ, 231, 594

Zank, G. P., Matthaeus, W. H., \& Smith, C. W. 1996, J. Geophys. Res., 101,17093

Zhou, Y., \& Matthaeus, W. H. 1990, J. Geophys. Res., 95, 14881 